\title{
Exercise induced depolarization changes in BSPMs for assessment of ischemic heart disease
}

\author{
Michał Kania ${ }^{1,2}$, Roman Maniewski ${ }^{1}$, Rajmund Zaczek ${ }^{3}$, Małgorzata Kobylecka ${ }^{4}$, Grzegorz Opolski ${ }^{3}$, \\ Leszek Królicki ${ }^{4}$ \\ ${ }^{1}$ Nalecz Institute of Biocybernetics and Biomedical Engineering (IBIB PAN), Warsaw, Poland \\ ${ }^{2}$ Electrophysiology and Heart Modeling Institute (IHU LIRYC), Bordeaux, France \\ ${ }^{3}$ I Chair and Department of Cardiology, Medical University of Warsaw, Warsaw, Poland \\ ${ }^{4}$ Department of Nuclear Medicine, Medical University of Warsaw, Warsaw, Poland
}

\begin{abstract}
We studied the effect of exercise-induced ischemia on high-resolution body surface potential maps in the depolarization interval. In particular our aim was to evaluate the effectiveness of selected nine ECG parameters in diagnostics of ischemic heart disease (IHD). The study group consisted of 123 subjects. The distributions of ECG signals averaged in time at rest and at peak exercise were compared. It was observed that for patients with IHD, averaged decreases of QRS amplitude at peak exercise were higher than in the control group and in the group of patients without IHD. IHD was associated also with enlargement of the area on the thorax surface where the decrease in the amplitude and increase in shape changes of the QRS complex were observed. The most effective parameters for the separation of patients with IHD were quantifiers of $Q R S$ shape changes. The use of an optimized ECG electrode layout improves the diagnostic value of $Q R S$ morphology descriptors.
\end{abstract}

\section{Introduction}

According to the estimates of the World Health Organisation, ischemic heart disease (IHD) was responsible for 7.4 million $(13.2 \%)$ of deaths globally in 2012 [1]. The problem needs to be resolved by proper education promoting healthy lifestyles and development of noninvasive and cost-effective methods for early diagnosis of cardiac diseases.

The 12-lead ECG exercise test is often used as a screening test for ischemic heart disease. However, its diagnostic value is limited [2]. Exercise-induced myocardial ischemia contributes to the generation of injury currents between differently perfused parts of myocardium. This potential gradient is inspected from the thorax surface with use of ECG electrodes 'looking' from different sides at the ischemic lesion in the heart. The limited number and coverage area of ECG leads used in standard ECG exercise testing could be in some cases the reason of IHD diagnostic failures. On the other hand the IHD pattern is searched mainly in the ST segment of the studied ECG, omitting its influence on the depolarization phase.

The aim of this study was to evaluate the effectiveness of multi-lead high-resolution ECG data analysis within the depolarization phase for IHD diagnostics. The exercise-induced ECG signal changes in the QRS complex were studied by applying nine ECG parameters and their performance was checked based on the results of IHD imaging methods: SPECT and coronarography.

\section{Materials and Methods}

The study was carried out in a group of 123 men i.e. 90 patients (age $61 \pm 9 \mathrm{yrs}$ ) and 33 healthy volunteers (age $55 \pm 15$ yrs). Subjects were recruited among clinically stable patients referred for myocardial perfusion SPECT imaging due to clinical suspicion of coronary artery disease: patients with a positive ECG exercise test, typical symptoms of angina pectoris, or patients with acute coronary syndrome with angiographically documented borderline coronary artery stenosis. Healthy volunteers did not have a history of cardiovascular disease and had normal resting and exercise 12-lead ECG. The myocardial perfusion was verified based on SPECT and coronarography results.

The high-resolution body surface potential maps (BSPMs) were recorded at rest and during exercise test performed on a supine ergometer with stepwise increasing load (by $25 \mathrm{~W}$ every $2 \mathrm{~min}$, beginning at $50 \mathrm{~W}$ ). The analysed data set included ECG signals from 67 active electrode locations on the thorax surface (Fig. 1). The signals were simultaneously acquired with $4096 \mathrm{~Hz}$ sampling frequency and digitized with 24-bit amplitude resolution. 
The ECG recordings were band-pass filtered with cutoff frequencies 0.05 and $250 \mathrm{~Hz}$. Baseline wander was reduced using a third-degree polynomial estimation and removal procedure. Temporally averaged ECG data sets from rest and peak exercise time frames were compared. Signal averaging was performed using a cross-correlation method for beat alignment. ECG characteristic points were automatically detected on the root-mean-square signal derived from all measured ECG leads, then were checked and corrected, if needed. Detailed descriptions of the data preprocessing are given in our previous work [3] [4].

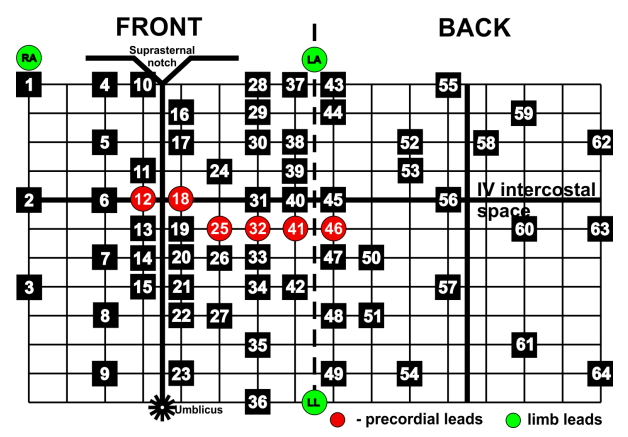

Figure 1. ECG electrode layout on the thorax surface

The nine ECG parameters were computed for each measured ECG lead to detect exercise-induced ischemic changes within the QRS complex.

Difference maps of QRS extreme amplitudes $\left(Q R S_{a}\right)$, mean potentials $\left(Q R S_{m}\right)$ and intervals $\left(Q R S_{\text {int }}\right)$ of QRS complex were determined according to relationship:

$$
\Delta X(i)=X^{e x}(i)-X^{\text {rest }}(i) \text {, }
$$

where: $X(i)$ - the value of parameter $X$ in the $i$-th ECG lead calculated from averaged ECG signals recorded at peak exercise (labeled 'ex') or measured at rest (labeled 'rest').

For detailed evaluation of the signal shape changes within the QRS complex the Distribution Function Method (DFM) [5] was used, which previously has been shown as a useful tool in detection of small variation in shape of chromatographic peaks [6] as well as in cardiac diagnosis $[7,8]$.

Let $s_{0}(t)$ be a reference signal measured at rest and $s_{j}(t)$ represents compared signal recorded at peak exercise. If $s_{0}(t)$ and $s_{j}(t)$ are equal in shape, signal $s_{j}(t)$ can be derived from $s_{0}(t)$ through an affine function:

$$
s_{j}(t)=k_{j} s_{0}\left(\frac{t-t_{j}}{a_{j}}\right) .
$$

The $k_{j}, a_{j}, t_{j}$ are respectively magnitude coefficient, scale coefficient, and delay. ECG signals are after baseline correction; therefore their offset was omitted in the above equation. The difference in shape between $s_{0}(t)$ and $s_{j}(t)$ could be characterized by function $\varphi$ defined by:

$$
S_{j}(t)=S_{0}(\varphi(t)) \text { i.e. } \varphi=S_{0}^{-1} \circ S_{j},
$$

where $S_{j}(t)$ and $S_{0}(t)$ are the normalized integral functions of $s_{j}(t)$ and $s_{0}(t)$ respectively, increasing from zero to one.
The shape variation between $s_{j}(t)$ and $s_{0}(t)$ can be quantified by a parameter $\delta$ measuring the distance between the function $\varphi$ and the least mean square line fitted on $\varphi$ :

$$
\delta=\sqrt{\frac{1}{M} \sum_{i=1}^{i=M}(\varphi(i)-y(i))^{2}} .
$$

The $\delta$ parameter describes the 'real' ECG morphology changes between a pair of two signals omitting the scaling effect i.e. stretching or shrinking of ECG waves either in amplitude or in time. The $\delta$ close to zero means that the waveform shape does not change, whereas $\delta$ greater than zero indicates appearance of additional ECG components like QRS complex shape change to $\mathrm{rSr}^{\prime}$ pattern.

Additionally, the standard parameters quantifying the ECG shape changes were computed: Pearson correlation coefficient $(R)$, and the root mean squared error (RMSE) between the reference signal $s_{0}(t)$ and the signal $s_{j}(t)$. The $R M S E$ parameter was normalized (NRMSE) to the range of amplitudes of the measurement data [9] in order to minimize the effect of inter-subject differences in the maximum values of signal amplitudes on the average RMSE values.

The multidimensional angle $\beta$ was calculated in order to compare the patterns of BSPMs of $Q R S_{a}, Q R S_{m}$ at rest and at peak exercise defined by the relationship

$$
\beta=\cos ^{-1}\left(\frac{x \circ y}{|x||y|}\right),
$$

where $x$ and $y$ are the vectors of ECG parameter values in all ECG leads at rest and at stress, respectively .

The selection of the studied subjects to the group of patients with and without myocardial ischemia was based on the SPECT and coronarography results. The assessment of statistical significance of differences between group means of ECG parameters values was performed using a nonparametric Mann-Whitney test and a p-value $<0.05$ was considered statistically significant.

\section{Results}

The changes in ECG parameter values due to exercise were observed. The degree and direction of these changes depends on the level of effort made on ergometer, as well as depends on the location of the ECG electrodes on the thorax surface, on chosen ECG parameter and on the group of studied subjects.

There were no significant changes in QRS duration during the exercise test. Mostly the QRS complex was shortened (in $67 \%$ of cases) rather than lengthened (in $33 \%$ of cases) in time. There were also no significant $\triangle Q R S_{\text {int }}$ differences between the studied groups.

On the right and the lower-left part of the thorax increases of the QRS amplitude prevailed whereas QRS amplitude decline was mainly observed in the upper-left part of the torso (Fig. 2). The QRS amplitude increased in BSPMs by an average of similar value in the groups of 
patients with IHD, without detected IHD and a group of healthy volunteers $(168 \mu \mathrm{V}, 138 \mu \mathrm{V}$ and $143 \mu \mathrm{V}$, respectively). However, the mean QRS amplitude decreases at peak exercise were greater in patients with IHD (Fig. 2,C) than those in a group of subjects without IHD (Fig. 2,B) and in a control group (Fig. 2,A): $356 \mu \mathrm{V},-163 \mu \mathrm{V}$ and $-127 \mu \mathrm{V}$, respectively. Myocardial ischemia was also associated with the enlargement of the area on the thorax surface where reductions towards the lower part of the chest of ECG signal amplitude were observed.

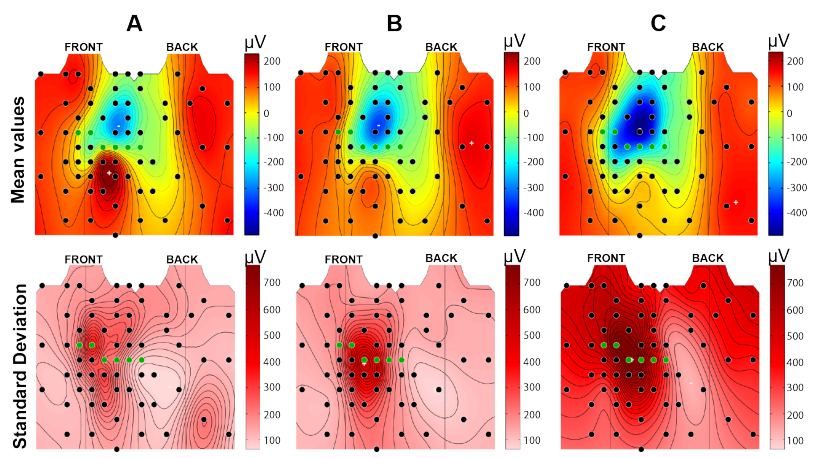

Figure 2. Mean BSPMs and corresponding standard deviation maps of $\triangle Q R S_{a}$ : A - in a group of healthy volunteers, B - in patients without IHD (negative SPECT result) and $\mathrm{C}$ - in patients with IHD (positive SPECT result).

Table 1. Mean changes \pm SD of studied ECG parameters at peak exercise with reference to those observed at rest for the control group and the group of the patients with a positive (+) and negative (-) SPECT result. Statistically significant $(\mathrm{p}<0.05)$ differences between the groups compared in adjacent columns are marked in bold.

\begin{tabular}{|c|c|c|c|c|c|}
\hline Param. & $\begin{array}{l}\text { Control } \\
\text { group }\end{array}$ & $\bar{p}$ & $\begin{array}{l}\text { Pts. } \\
(+)\end{array}$ & $\overline{\mathbf{p}}$ & $\begin{array}{l}\text { Pts. } \\
(-)\end{array}$ \\
\hline $\begin{array}{l}\Delta Q R S_{a}{ }^{T} \\
{[\mu \mathrm{V}]}\end{array}$ & $\begin{array}{l}-491 \\
\pm 208\end{array}$ & .28 & $\begin{array}{l}-776 . \\
\pm 889 .\end{array}$ & .088 & $\begin{array}{l}-511 . \\
\pm 409 .\end{array}$ \\
\hline $\begin{array}{l}\beta Q R S_{a} \\
\left.{ }^{0}\right]\end{array}$ & $\begin{array}{l}8.64 \\
\pm 2.31\end{array}$ & .427 & $\begin{array}{l}12.31 \\
\pm 1.30\end{array}$ & .153 & $\begin{array}{l}9.47 \\
\pm 8.54\end{array}$ \\
\hline $\begin{array}{l}\qquad Q R S_{m}{ }^{h} \\
{[\mu \mathrm{V}]}\end{array}$ & $\begin{array}{l}137 \\
\pm 60\end{array}$ & .003 & $\begin{array}{l}113.5 \\
\pm 90.5\end{array}$ & .289 & $\begin{array}{l}108 \\
\pm 53\end{array}$ \\
\hline $\begin{array}{l}\beta Q R S_{m} \\
{\left[{ }^{\circ}\right]}\end{array}$ & $\begin{array}{l}13.24 \\
\pm 5.23\end{array}$ & .014 & $\begin{array}{l}11.01 \\
\pm 5.95\end{array}$ & .213 & $\begin{array}{l}11.8 \\
\pm 6.12\end{array}$ \\
\hline $\begin{array}{l}\Delta Q R S_{i n t}{ }^{l} \\
{[\mathrm{~ms}]}\end{array}$ & $\begin{array}{l}-7.73 \\
\pm 5.90\end{array}$ & .4 & $\begin{array}{l}-8.85 \\
\pm 8.39\end{array}$ & .46 & $\begin{array}{l}-8.49 \\
\pm 6.80\end{array}$ \\
\hline $\begin{array}{l}\delta \\
{[\mathrm{ms}]}\end{array}$ & $\begin{array}{l}2.34 \\
\pm 2.45\end{array}$ & .200 & $\begin{array}{l}3.42 \\
\pm 3.76\end{array}$ & .042 & $\begin{array}{l}2.15 \\
\pm 2.91\end{array}$ \\
\hline$R$ & $\begin{array}{l}1.00 \\
\pm .004\end{array}$ & .37 & $\begin{array}{l}0.97 \\
\pm 0.08\end{array}$ & .040 & $\begin{array}{l}0.99 \\
\pm 0.05\end{array}$ \\
\hline $\begin{array}{l}\text { RMSE } \\
{[\mu \mathrm{V}]}\end{array}$ & $\begin{array}{l}281.5 \\
\pm 82\end{array}$ & .16 & $\begin{array}{l}383.5 \\
\pm 73.5\end{array}$ & .092 & $\begin{array}{l}299 \\
\pm 164\end{array}$ \\
\hline $\begin{array}{l}\text { NRMSE } \\
{[\%]}\end{array}$ & $\begin{array}{l}30 \\
\pm 7\end{array}$ & 0.40 & $\begin{array}{l}31 \\
\pm 9\end{array}$ & 0.24 & $\begin{array}{l}30 \\
\pm 9\end{array}$ \\
\hline
\end{tabular}

Table 1 shows the results of the statistical analysis of nine computed electrocardiographic parameters. Their group mean values were calculated from individual BSPMs, separately for maximal and minimal values in the maps. They correspond to the greatest increase (labeled " $h$ "), or the greatest decrease (labeled " $l$ ") of the ECG parameter at peak exercise.

Significant differences in QRS morphology changes between the groups of patients without IHD (negative SPECT result) and those with IHD (positive SPECT result) were found. This is particularly the difference in values of $\delta$ and $R$ given in Table 1. The observed differences may be a result of a 'real' change in QRS morphology - not due to amplitude scaling, as evidenced by no significant differences found in values of NRMSE.

The sensitivities and specificities for the adopted decision model were determined to get more accurate assessment of the performance of analyzed parameters in the IHD diagnosis. The results are shown in Table 2. The given decision thresholds (DT) were determined from the ROC analysis at the intersection point of the sensitivity and specificity curves plotted against the cut-off parameter values. The test result was considered positive (ischemia detected) when in at least one of the ECG leads the parameter value was greater or equal to the decision threshold for $\delta$, and less or equal to the DT for $R$.

Table 2. The values of sensitivity and specificity in IHD detection evaluated with respect to: a group of patients with positive result of SPECT (SPECT + ) or a group of patients with positive results of both SPECT and coronarography (SPECT + Coro + ). The specificities were computed with respect to: a control group, patients with a negative SPECT result or a group of patients with negative results of both reference studies (SPECT-Coro-).

\begin{tabular}{llll}
\hline Groups & $\begin{array}{l}\text { SPECT+/ } \\
\text { Healthy }\end{array}$ & $\begin{array}{l}\text { SPECT+/ } \\
\text { SPECT- }\end{array}$ & $\begin{array}{l}\text { SPECT+Coro+/ } \\
\text { SPECT-Coro- }\end{array}$ \\
\hline$\underline{\boldsymbol{\delta}}$ & & & \\
Sensitivity & $54.2 \%$ & $56.2 \%$ & $62.9 \%$ \\
Specificity & $52.9 \%$ & $57.1 \%$ & $62.5 \%$ \\
DT & $\geq 1.62$ & $\geq 1.56$ & $\geq 1.81$ \\
AUC & 0.55 & 0.6 & 0.57 \\
\hline$\underline{\boldsymbol{R}}$ & & & \\
Sensitivity & $54.2 \%$ & $54.2 \%$ & $62.9 \%$ \\
Specificity & $52.9 \%$ & $54.8 \%$ & $62.5 \%$ \\
DT & $\leq 0.33$ & $\leq 0.34$ & $\leq 0.27$ \\
AUC & 0.51 & 0.54 & 0.61 \\
\hline DT - decision threshold, AUC - area under ROC curve
\end{tabular}

\section{Discussion and conclusion}

In this study significant exercise-induced changes of QRS complex morphology were observed (Table 1). The best performance in the detection of electrocardiographic 
signs of myocardial ischemia within the depolarization period was shown by the $\delta$ and $R$ parameters.

Specific ischemic changes that arise as a result of coronary artery occlusion were studied earlier in the maps of the QRS complex [10, 11]. Spekhorst et al. [10] analysed the ECG signals from 62 leads before, during, and after PTCA. They found specific, temporary changes in QRS amplitudes that occur during balloon inflation in the widened coronary artery. These temporary changes in the ECG signal were not visible in the average amplitudes of the QRS complex. Our study also showed no significant differences in the average potentials of the QRS complex $\left(\triangle Q R S_{m}\right.$, Table 1) between the group of patients with positive SPECT and a group of patients with a negative SPECT results. However, obtained results indicated significant changes in parameters assessing exercise-induced changes in QRS morphology $(\delta, R$, Table 1), in particular detecting the temporal variations in the ECG signal. As hypothesized by Spekhorst et al. [10], the temporal changes in the amplitude of the QRS complex that appear during myocardial ischemia may be associated with delayed conduction within ischemic tissue.

Our relatively low values of sensitivity and specificity (Table 2) may results from the use of one simple decision threshold for all ECG electrode positions. Another reason for the low diagnostic value could be the usage of electrode locations where the recorded ECG signal has low amplitude being more sensitive to interference in comparison to the electrodes located close to the signal source. Exercise-induced QRS shape changes are different depending on the location of the measurement electrodes. For this reason, the use of one decision threshold may cause incorrect classification of subjects. The selection of a subset of ECG leads covering areas of the torso where the greatest changes in ECG morphology were noticed may be more appropriate in this case. To find that out the sensitivity and specificity of $\delta$ and $R$ were computed for eight optimal ECG lead locations. The mean BSPMs of $\delta$ and $R$ in the groups of patients SPECT + Coro + and SPECT-Coro- (see description under Table 2) were compared. The optimal ECG lead subsets were chosen by taking the six first lead positions characterized by the greatest intergroup differences (i.e. with the lowest p-values). The sensitivities/specificities for such optimized ECG lead layouts were $63 \% / 62 \%$ and $74 \% / 75 \%$ for $\delta$ and $R$ respectively. It means that even by applying such a simple method for ECG electrode layout optimization one can reach the same $(\delta)$ or even better $(R)$ levels of sensitivity and specificity as in case of analysis of all ECG leads. For IHD patients the changes in amplitude and ECG morphology were found in ECG electrodes located outside the area of precordial leads. This may suggests that the standard ECG lead system is not the optimal for exercise assessment of IHD and could be improved. Seeking the optimal number and location of
ECG leads for the analysis of QRS morphology changes or optimal, ECG lead specific diagnostic thresholds could be the subject of future studies.

\section{Acknowledgements}

This work was financially supported by National Science Centre of Poland (grant no. 2011/01/N/ST7/06690 and grant no. NN 518504 339).

\section{References}

[1] WHO. The top 10 causes of death: Fact sheet $\mathrm{N}^{\circ} 310$. World Health Organisation, 2013. Available: http://www.who.int/mediacentre/factsheets/fs310/en.

[2] Finlay DD, Nugent CD, Kellett JG, et al. Synthesising the 12-lead electrocardiogram: Trends and challenges. European Journal of Internal Medicine, vol. 18, no. 8. pp. 566-570, 2007.

[3] Kania M, Maniewski R, Kobylecka M, et al. Prognostic value of the total cosine $R$ to $T$ measured in high resolution body surface potential mapping during exercise test. Biomed. Signal Process. Control, vol. 20, pp. 135141, 2015.

[4] Fereniec M, Stix G, Kania M, et al. Risk assessment of ventricular arrhythmia using new parameters based on high resolution body surface potential mapping. Med. Sci. Monit., vol. 17, no. 3, pp. MT26-MT33, 2011.

[5] Rix H, Malengé JP. Detecting small variations in shape. Syst. Man Cybern. IEEE Trans., vol. 10, no. 2, p. IEEETransactions, 1980.

[6] Rix H. Detection of small variations in shape between two chromatographic peaks," J. Chromatogr. A, vol. 204, pp. 163-165, 1981.

[7] Fereniec M, Maniewski R, Karpinski G, et al. Highresolution multichannel measurement and analysis of cardiac repolarization. Biocybern. Biomed. Eng., vol. 28, no. 3, pp. 61-69, 2008.

[8] Khaddoumi B, Rix H, Meste O, et al. Body surface ECG signal shape dispersion. IEEE Trans. Biomed. Eng., vol. 53, no. 12, pp. 2491-2500, 2006.

[9] Kania M, Rix H, Fereniec M, et al. The effect of precordial lead displacement on ECG morphology. Med. Biol. Eng. Comput., vol. 52, no. 2, pp. 109-119, 2014.

[10] Spekhorst H, SippensGroenewegen A, David GK, et al. Body surface mapping during percutaneous transluminal coronary angioplasty. QRS changes indicating regional myocardial conduction delay. Circulation, vol. 81 , no. 3 , pp. 840-849, 1990.

[11] Préda I, Nadeau R, Savard P, et al. QRS alterations in body surface potential distributions during percutaneous transluminal coronary angioplasty in single-vessel disease. J. Electrocardiol., vol. 27, no. 4, pp. 311-322, 1994.

Address for correspondence:

Michal Kania

Nalecz Institute of Biocybernetics and Biomedical Engineering, Laboratory for Bioelectromagnetical Measurements and Imaging ul. Trojdena 4, 02-109 Warsaw, Poland. 